\title{
Optimal operation of water pumping stations
}

\author{
D. Al-Ani \& S. Habibi \\ Mechanical Engineering Department, McMaster University, Hamilton, \\ ON, Canada
}

\begin{abstract}
In water distribution applications, energy cost is one of the most important cost components in which large amounts of electricity are required to pump, transport, and supply water to both domestic and businesses districts. These applications are heavily dependent upon energy costs. Although, there are many optimization methodologies that can theoretically maximize energy cost savings, but there are none (yet) that have practically succeeded. Therefore, the goal of the new optimization strategies should not be only to solve the energyoptimization problems, but also to have constraints criteria that seek for the design and management of the water distribution systems. Some of these strategies can be used: (a) to improve the operation of pumping stations for a given water demand; (b) to include a regulating reservoir between the water supply source and the delivery system; and (c) to control water level in tanks and reservoir to prevent overflow and draining. Such initiatives are likely to be more successful if they involve a role of maintaining the safety and the reliability of the pumping station while optimizing its operation. A novel strategy that can result in minimum operation costs and can lead to less energy consumption and then utility bills is proposed in this paper. In this strategy, a new heuristic algorithm, referred to as the Adaptive Parallel Multi-objective Differential Evolution (AP-MODE), is developed and used for: (a) selecting the lowest cost or optimum pump combinations (i.e., optimal pump scheduling) in water distribution systems; (b) evaluating the total energy costs required to operate the network; and (c) determining the reservoir storage capacity (i.e., optimal reservoir operation) to store water that is pumped during peak-off hours to be used during peak periods. This strategy is applied to the water distribution system of one rural district located in Saskatoon City, Saskatchewan, Canada.
\end{abstract}


The analysis indicated that the optimal operation of pump groups and regulating reservoirs are energy cost effective.

Keywords: pumping stations, water distribution systems, energy-efficiency, heuristic algorithms.

\section{Introduction}

Power rates for Europe's Water Industry are up $10 \%$ in the last decade and expected to increase more in the future. The consequence of this higher power costs will drive to the dilemma of boosting wholesale power rates at least $15 \%$ in 2035. This increment in the energy prices has created the need for increased emphasis on efficient energy use. In many water distribution systems, due to large amounts of energy are required to pump, transport, and supply water, improved energy-efficiency of pumping stations will lead to a significant reduction in energy costs. Therefore, energy-efficiency in water distribution applications must be regarded as a priority when more efficient system operation is sought.

The overall efficiency of a water distribution system can be motivated by applying a set of operational regulations to storage tanks to narrow the temporal gaps between supply and demand [1-4]. This in turns will evidently enhance the degree of freedom in water consumers and also make the system more flexible to dynamically adapt upon changes in water demand or sudden event that require a certain volume of water (i.e., fire or damage in a pipeline scenarios). Therefore, in a water distribution system, the control of a reservoir or tank's operation reduces the overall operational cost as well as improves its reliability. Tanks are used for storing water that is pumped from resources during off-peak period (i.e., low-energy tariff) and used for supplying water to consumers during peak electrical demand (i.e., high-energy tariff) [5].

This paper presents the development and application of a new energyefficiency approach that considers the aforementioned difficulties. The objective is to optimize the operation of both pumping stations and reservoirs while satisfying water demands and system hydraulic requirements, and therefore, to have impacts on the optimal status of energy costs (i.e., in terms of pump combinations) at water pumping stations, water quantity (i.e., in terms of volume of delivered water), system reliability (in terms of storage capacity and water level in reservoirs), and system safety (i.e., in terms of maintaining the mass balance between the pump discharge and water demand). And all these types of energy-efficiency depend on directly the energy consumed by pumps and indirectly on the water level maintained in reservoirs. It should be noted that the determination of optimum pump scheduling and reservoir operation are of interest in the model developed. Integration of the operation schedule with the hydraulic model in an optimization method is the main purpose of this paper. Then, the energy-efficiency approach is verified by applying the developed optimization strategy to an existing water distribution systems in a rural district located in Saskatoon, province of Saskatchewan, Canada. 
The paper is organized as follows: an introduction of optimization methods for water pumping stations and reservoirs are provided in Section 1. The formulation of the energy-problem (i.e., objective function) is given in Section 2. In Section 3, the layout and the hydraulic model analyses for a rural water distribution system are performed and presented. The new Adaptive Parallel Multi-objective Differential Evolution (AP-MODE) is thoroughly explained in Section 4. The proposed methodology is applied to an existing rural water distribution system located in Saskatoon, province of Saskatchewan, Canada as described in Section 5. In Section 6, a simulation set of solutions for the optimal pump scheduling and reservoir operation over a long period of time is tabulated, presented, and then discussed. Finally, the remarks of the proposed work are concluded in Section 7.

\section{Formulation of energy-problem}

The energy-problem (i.e., objective function) described in this paper minimizes the total electricity costs that are including both electricity cost and maximum demand charge (i.e., CAD\$), maintenance costs, system reliability, and system safety.

where:

$$
\text { Minimize } y=\left(f_{1}(x), f_{2}(x), f_{3}(x), f_{4}(x), f_{5}(x)\right)
$$

$f_{1}$ : electrical energy cost; and is defined as the cost of electrical energy consumed by all pumps of the pumping stations during the optimization period $[6,7]$ (see eqn. (2)),

$f_{2}$ : maximum demand charge; and is defined as an additional charge (i.e., an expensive charge) added when the clients exceed their maximum allowable electric energy (see eqn. (3)),

$f_{3}$ : maintenance costs; and is defined as the total number of times pumps are switches ON at every time interval (see eqn. (4)),

$f_{4}$ : network reliability; and is defined as the ability of the system to provide service at an acceptable level in spite of abnormal conditions and can be mapped into the reservoir level variation $(\Delta h)$ between the beginning and the end of the optimization period (see eqn. (5)),

$f_{5}$ : network safety; and $\mathrm{s}$ defined as the system mass balance, the volume of water, that is the difference between the pump discharge and the water demands of the delivery system at every time interval (see eqn. (6)),

$x \in X \subset B^{168 . N p}$ is the decision vector, $B=\{0,1\}$, (where $N P$ is number of pumps) and

$y=\left(y_{1}, y_{2}, y_{3}, y_{4}\right) \in Y \subset \mathbb{R}^{4}$ is the objective vector.

Constraints on the optimization problem define the criteria that classify the solutions, obtained by the optimization algorithm, whether they are feasible. A solution is called feasible if it satisfies all problem constraints. Likewise, an infeasible solution is defined as a solution to an optimization problem that does not satisfy all or potentially any of the constraints or not feasible in order to define the system performance. Constraints may be defined as operational and boundary conditions for a given energy-optimization problem. The mathematical 
representation of the energy-problem (i.e., cost function) as well as the associated constraints is given as follows.

- $\quad$ Cost function

$$
\begin{gathered}
f_{1}=\sum_{i=1}^{T p} \underbrace{\left[T_{r} E C\left[P_{C}(i)\right]\right]}_{\text {Electrical Energy Cost }} \\
f_{2}=\sum_{i=1}^{T p} \underbrace{\max [P(P c(i))]}_{\text {Maximum Demand Charge }} \\
f_{3}=\sum_{i=1}^{T p} \underbrace{|\max (0 ; P c(i)-P c(i-1))|}_{\text {Maintenance Costs }} \\
f_{4}=\Delta h=\underbrace{\sum_{i=1}^{T p}\left[D\left(P c_{i}\right)-\sum_{k=1}^{N T} \frac{d_{i, k}}{S_{k}}\right]}_{\text {System Reliability }} \\
f_{5}=\sum_{\text {System Safety }}^{\sum_{i=1}^{T p}\left[D(P c(i)) \sum_{k=1}^{N D} \text { Demand }(i, k)\right] \frac{\Delta i}{F}}
\end{gathered}
$$

- $\quad$ Subject to

1. Pressure constraints at a junction node:

$$
\operatorname{Pmin}_{j} \leq P_{j}(i) \leq P \max _{j} \quad, \quad \forall j \forall i
$$

2. Water level constraints in a tank:

$$
\begin{gathered}
h_{j}(i)=h_{j}(i-1)+\frac{D(P c(i))_{j}-d_{j}(i)}{S_{j}} \\
h_{j}(i) \geq \text { hmin }_{j}, \quad \forall j \forall i \\
h_{j}(i) \leq \text { hmax }_{j}, \quad \forall j \forall i
\end{gathered}
$$

3. Pump switching at a water pumping station:

$$
S W_{k} \leq S W \max _{k}, \forall k
$$

where:

bp

is billing period

$D\left(P c_{i}\right)$

is discharge pumped at time interval $(i)$ using pump combination $P c$

$\operatorname{Demand}(i, k)$

$d_{i, k} \quad$ is water enter the tank at period $(i)$ for tank $(k)$

$E C$

interval $(i)(\mathrm{kW} / \mathrm{h})$

$E_{b p}^{j} \quad$ is max. energy consumption during the billing period ( $\left.b p\right)$

$h_{j}(i) \quad$ is the estimated water level of tank $j$ at time $i$ 


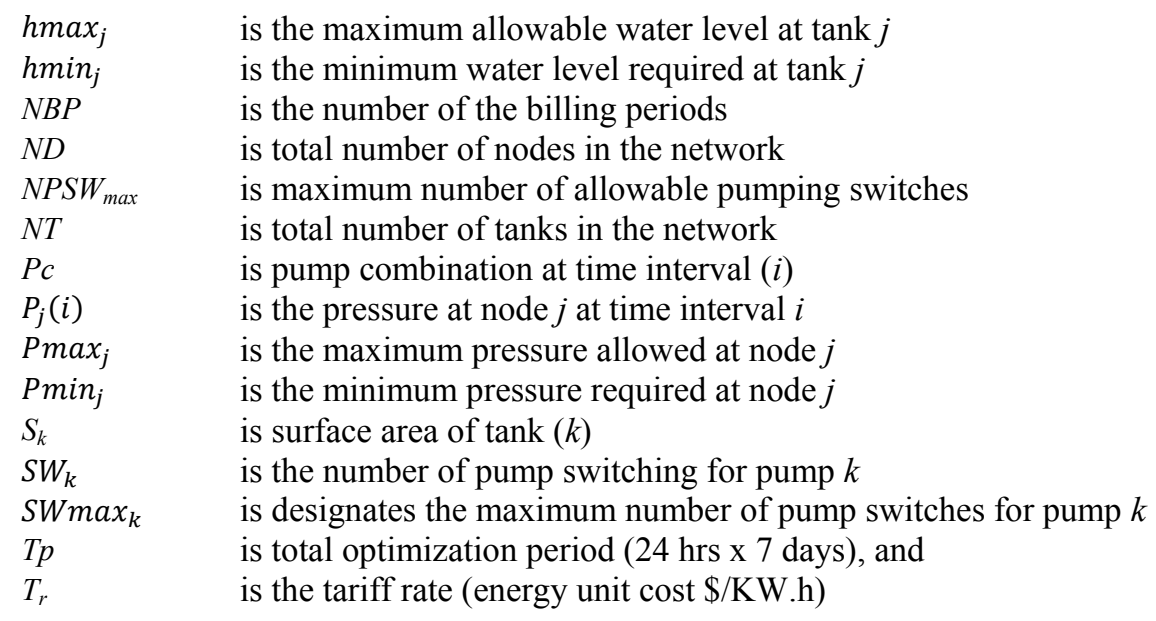

Discrete hourly-base intervals is adopted for the optimization period because the demands display a pronounced daily cycle, and energy tariffs are based on time of day.

\section{System layout and hydraulic model analysis}

Saskatoon West water distribution system (WDS) infrastructure, like any other rural distribution system, consists of arterial pipes, junction nodes, control valves, reservoirs, tanks, primary lifting pumping stations (main and boosting stations), hydrants, main-line meters, service connections, and backflow preventers at man station. The system draws water from Saskatoon River and many other wells. A simple schematic diagram of this system is shown below.

Saskatoon West WDS supplies water to five major clients as follows: (see Table 1).

Table 1: $\quad$ Major clients in Saskatoon West water distribution system.

\begin{tabular}{lll}
\hline Name & Customer Type & Supplied Water \\
\hline Client 1 & Residential & Raw water \\
\hline Client 2 & Residential & Treated water \\
\hline Client 3 & Industrial & Raw water \\
\hline Client 4 & Industrial & Raw water \\
\hline Client 5 & Industrial & Raw water \\
\hline
\end{tabular}

The Saskatoon West WDS contains 169 pipes, 153 junction nodes, 6 pressure reducing valve, one treatment plant, four elevated and grounded storage tanks, 5 variable-speed pumps located at main and boosting pumping stations.

In optimizing pump operation in a water distribution system, a simple model can be used because only relatively large mains between pumps, reservoirs, and 


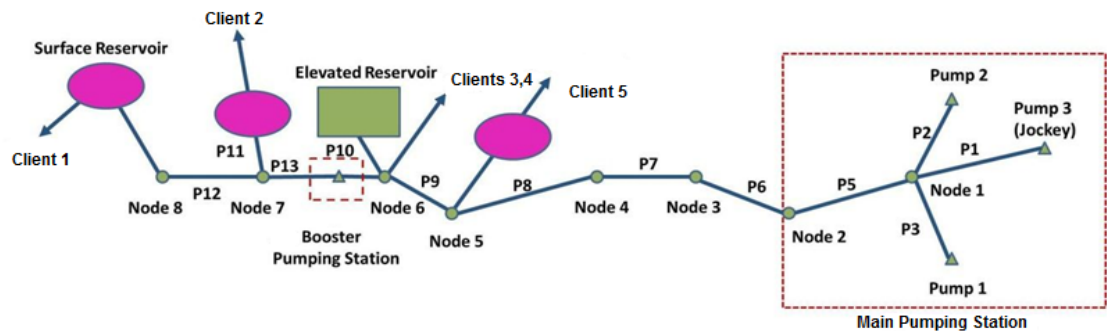

Figure 1: Simple schematic diagram of Saskatoon West water distribution system.

junction nodes are important in calculations [8]. Therefore, in this work, a simplified hydraulic model based on a real-world water distribution system in Saskatoon is considered. Figure 2 shows the schematic model using five pumps installed in two pumping stations and has many grounded and elevated reservoirs. In

pumping stations, pumping capacities are assumed during the time interval, and therefore, each pump combination has a fixed maximum flow rate, electrical energy consumption, and maximum power. Table 2 presents the characteristics of a pump combination for the Saskatoon West WDS model.

Table 2: $\quad$ Technical characteristics of pump combinations.

\begin{tabular}{cccc|cccc}
\hline $\begin{array}{c}\text { Pump } \\
\text { Combination }\end{array}$ & $\begin{array}{c}\text { Code } \\
\text { P1...P5 }\end{array}$ & $\begin{array}{c}\text { Max. } \\
\text { Flow } \\
\text { (IGPM) }\end{array}$ & $\begin{array}{c}\text { Power } \\
\text { (KW) }\end{array}$ & $\begin{array}{c}\text { Pump } \\
\text { Combination }\end{array}$ & $\begin{array}{c}\text { Code } \\
\text { P1...P5 }\end{array}$ & $\begin{array}{c}\text { Max. } \\
\text { Flow } \\
\text { (IGPM) }\end{array}$ & $\begin{array}{c}\text { Power } \\
(\text { KW })\end{array}$ \\
\hline 0 & 00000 & 0 & 0 & 16 & 10000 & 922 & 108 \\
\hline 1 & 00001 & 1372 & 116 & 17 & 10001 & 2294 & 224 \\
\hline 2 & 00010 & 1372 & 116 & 18 & 10010 & 2294 & 224 \\
\hline 3 & 00011 & N/A & N/A & 19 & 10011 & N/A & N/A \\
\hline 4 & 00100 & 198 & 12 & 20 & 10100 & 1120 & 120 \\
\hline 5 & 00101 & 1570 & 128 & 21 & 10101 & 2492 & 336 \\
\hline 6 & 00110 & 1570 & 128 & 22 & 10110 & 2492 & 336 \\
\hline 7 & 00111 & N/A & N/A & 23 & 10111 & N/A & N/A \\
\hline 8 & 01000 & 922 & 108 & 24 & 11000 & 1844 & 216 \\
\hline 9 & 01001 & 2294 & 224 & 25 & 11001 & 3116 & 332 \\
\hline 10 & 01010 & 2294 & 224 & 26 & 11010 & 3116 & 332 \\
\hline 11 & 01011 & N/A & N/A & 27 & 11011 & N/A & N/A \\
\hline 12 & 01100 & 1120 & 120 & 28 & 11100 & N/A & N/A \\
\hline 13 & 01101 & 2492 & 336 & 29 & 11101 & N/A & N/A \\
\hline 14 & 01110 & 2492 & 336 & 30 & 11110 & N/A & N/A \\
\hline 15 & 01111 & N/A & N/A & 31 & 11111 & N/A & N/A \\
\hline
\end{tabular}

* N/A stands for not applicable due to system's regulation. 


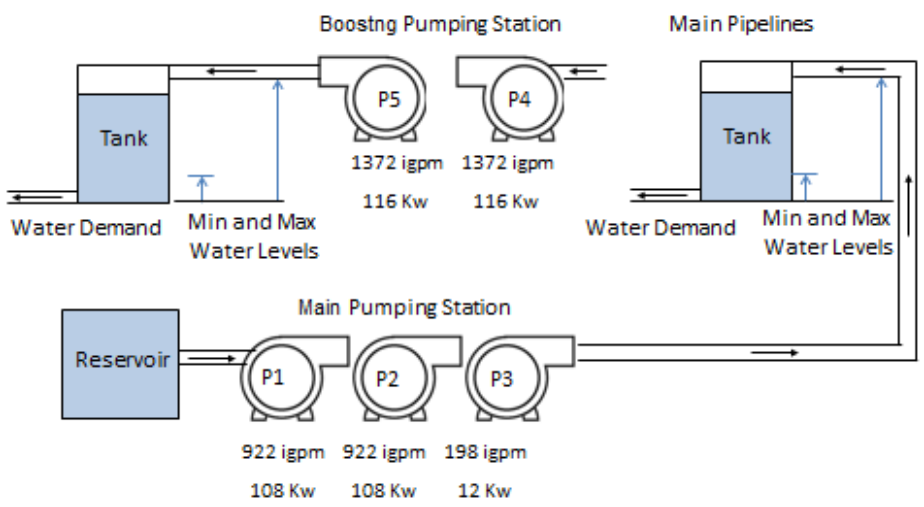

Figure 2: $\quad$ Schematic of simplified water distribution model.

The simulation results of this model are obtained and presented in the following figures.

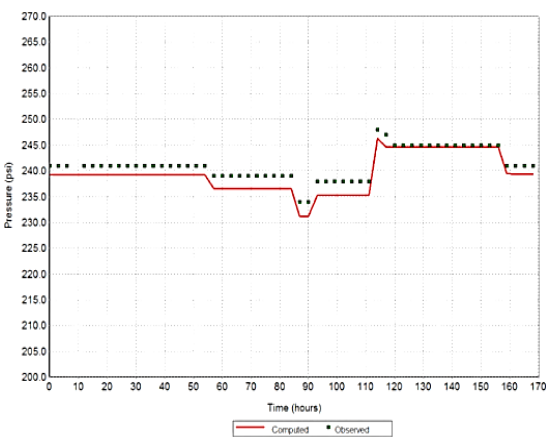

Figure 3: $\quad$ Pressure at Node 1 (see Figure 1).

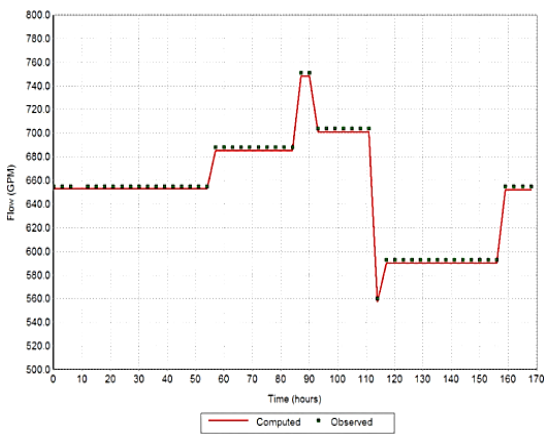

Figure 4: Flow at Link 11 (see Figure 1).

The outcome obtained from the analysis of the hydraulic model indicates that the model shows very good matches between the observed profile and the computed ones in terms of energy costs, pressure, and flow profiles.

\section{Adaptive parallel multi-objective differential evolution}

Differential Evolution (DE) is first proposed by Price and Storn in 1997 [9]. Like other Evolutionary Algorithms (EAs), DE is a population-based and stochastic algorithm that employs a group of potential solutions, called as individuals, to explore and then exploit the search-space in order to converge into a global optimum. As an iterative-based algorithm, the evolution of these individuals occurs by generating a new offspring using combinations of randomly chosen individuals from the population. In this context, the operation of producing new 
Table 3: Differential evolution mutation strategies.

\begin{tabular}{|c|c|c|}
\hline & DE $/ x / y / z$ Strategy & Interpretation \\
\hline 1. & $\mathrm{DE} /$ best/1/exp & $\begin{array}{l}\text { The best vector to be perturbed, } \\
\text { - } \quad \text { One vector considered for perturbation of best } \\
\text { vector, and } \\
\text { - Exponential type of crossover. }\end{array}$ \\
\hline & $\mathrm{DE} / \mathrm{rand} / 1 / \exp$ & $\begin{array}{l}\text { - The random vector to be perturbed, } \\
\text { One vector considered for perturbation of } \\
\text { random vector, and } \\
\text { - Exponential type of crossover. }\end{array}$ \\
\hline & $\mathrm{DE} /$ rand-to-best/1/exp & $\begin{array}{l}\text { - The random to best vector to be perturbed, } \\
\text { - One vector considered for perturbation of } \\
\text { random to best vector, and } \\
\text { - Exponential type of crossover. }\end{array}$ \\
\hline 4. & $\mathrm{DE} /$ best/2/exp & $\begin{array}{l}\text { - The best vector to be perturbed, } \\
\text { - Two vectors considered for perturbation of best } \\
\text { vector, and } \\
\text { - Exponential type of crossover. }\end{array}$ \\
\hline 5. & $\mathrm{DE} / \mathrm{rand} / 2 / \exp$ & $\begin{array}{l}\text { - The random vector to be perturbed, } \\
\text { Two vectors considered for perturbation of } \\
\text { random vector, and } \\
\text { - Exponential type of crossover. }\end{array}$ \\
\hline & $\mathrm{DE} /$ best/1/bin & $\begin{array}{l}\text { - The best vector to be perturbed, } \\
\text { - One vector considered for perturbation of best } \\
\text { vector, and } \\
\text { - Binomial type of crossover. }\end{array}$ \\
\hline 7. & $\mathrm{DE} / \mathrm{rand} / 1 / \mathrm{bin}$ & $\begin{array}{l}\text { The random vector to be perturbed, } \\
\text { One vector considered for perturbation of } \\
\text { random vector, and } \\
\text { Binomial type of crossover. }\end{array}$ \\
\hline & $\mathrm{DE} /$ rand-to-best/1/bin & $\begin{array}{l}\text { - The random to best vector to be perturbed, } \\
\text { - One vector considered for perturbation of } \\
\text { random to best vector, and } \\
\text { - } \quad \text { Binomial type of crossover. }\end{array}$ \\
\hline 9. & $\mathrm{DE} /$ best/2/bin & $\begin{array}{l}\text { - The best vector to be perturbed, } \\
\text { - Two vectors considered for perturbation of best } \\
\text { vector, and } \\
\text { - Binomial type of crossover. }\end{array}$ \\
\hline 10. & $\mathrm{DE} / \mathrm{rand} / 2 / \mathrm{bin}$ & $\begin{array}{l}\text { - The random vector to be perturbed, } \\
\text { - Two vectors considered for perturbation of } \\
\text { random vector, and } \\
\text { - Binomial type of crossover. }\end{array}$ \\
\hline
\end{tabular}

individuals can be referred to as mutation. Hence, DE can be seen as an algorithm that combines simple arithmetic operators with the classical schemes of crossover, mutation, and selection. In 2005, Price and Storn proposed ten different mutation strategies of DE (as presented in Table 3) [10]. Each strategyis found to work well for a given problem and may not work out best when applied 
for a different problem. However, among these ten strategies, strategy 7 is found to be the most successful and the most widely used mutation strategy.

\subsection{Proposed algorithm}

In this paper, we are presenting a new Adaptive Parallel Multi-objective Differential Evolution (AP-MODE) algorithm to be used a solver for the energyefficiency approach by which a constrained multi-objective optimization problem, referred to as energy-problem, is evaluated (at every iteration) to find an optimal Pareto set of solutions (i.e., known as Pareto-front). The AP-MODE algorithm is described in the following subsections.

\subsubsection{New adaptive techniques}

In this work, a new self-directed adaptive technique is proposed. In this technique, the adaptivity and refinement are applied to most of the control parameters, namely: crossover constant and scaling factor.

Based on the DE model, the scaling factor and crossover constant (referred to as $F$ and $C R$, respectively) are very important for the exploration and exploitation abilities of the proposed AP-MODE algorithm. According to the effect of these two parameters, the proposed AP-MODE dynamically adjusts their values in relation to four conditions as provided in Table 4. Therefore, the self-adjustments of those coefficients can lead the APC-MODE algorithm to produce high-quality Pareto-front solutions.

Table 4: The strategies for tuning the scaling factor and crossover constant.

\begin{tabular}{llccc}
\hline Cases & Durations & States & $\boldsymbol{C R}$ & $\boldsymbol{F}$ \\
\hline Case 1 & At the beginning & Exploration & Larger & Smaller \\
\hline Case 2 & At the end & Exploitation & Smaller & Larger \\
\hline Case 3 & When landed-in & Convergence & Decreased & Increased \\
\hline Case 4 & When stagnating & Jumping-out & Increased & Decreased \\
\hline
\end{tabular}

The four strategies (i.e., the cases outlined in the above table) are listed according to the evolutionary states of the search process. The value of $C R$ is initialized with a reasonably large value and gradually changes in the exploration state, while a larger $F$ is required in the convergence state. Along with the search algorithm, a balance between global and local search ability is adaptively attained. Moreover, in the case of getting trapped into a local optimum (i.e., in the jumping-out state), larger $C R$ with a smaller $F$ are required to make the population separate from the local region and fly to the new and better region. Accordingly, the scaling factor and crossover constant are dynamically changing their values according to eqns. (11) and (12):

$$
C R=C R_{\max }-\left(C R_{\max }-C R_{\min }\right) \times e^{-\left(5 \times \frac{\text { iter }}{\text { Maxiter }}\right)^{2 *}}
$$




$$
F=F_{\text {min }}+\left(F_{\text {max }}-F_{\text {min }}\right) \times e^{-\left(5 \times \frac{\text { iter }}{\text { Maxiter }}\right)^{2 * *}}
$$

${ }^{*}$ The exponential term value varies within the range of [1, 0.1], 1 at the first iteration, and 0.1 at the last iteration.

${ }^{* *}$ The exponential term value varies within the range of $[0.1,1], 0.1$ at the first iteration, and 1 at the last iteration.

where $C R_{\max }, C R_{\min }, F_{\max }$, and $F_{\max }$ are the upper and lower bounds of crossover constant and scaling factor, respectively. And Maxiter is the total number of iterations.

\subsubsection{Parallel Islands model}

A common use of the decomposition approach is referred to as the Parallel Islands. In this approach, the whole population is divided into several subpopulations. Each sub-population is assigned to a different processor (island). Each processor runs a sequential MODE on its population. Parallel islands models allow migration (i.e. periodic exchange) of good candidate solutions from one island to another after every fitness evaluation [11]. This is done by promoting information sharing; the best individual of each subpopulation is migrated to other subpopulations, according to a predefined topology. The topology of the proposed scheme is a ring, i.e. the best individuals from each subpopulation are allowed only to migrate to the next subpopulation of the ring. Semi-isolated sub-populations help maintain MODE diversity. Therefore, the swarm of each island can explore a different part of the search space. Thereby parallel islands models allowing MODE algorithms to efficiently solve the multiobjective optimization problems.

In this work, the parallel islands model is implemented using the Parallel Computing toolbox and MATLAB Distributed Computing Server. The toolbox allows solving computationally problems using MATLAB ${ }^{\circledR}$ on multi-core and multiprocessor computers. The parallel computing toolbox consists such that it implements task-parallel and data-parallel algorithms without programming for specific hardware architecture. The toolbox also performs the execution of multitask job by evaluating each of its tasks and returning the results. Moreover, the parallel computing toolbox allows up to eight MATLAB workers to run on a local machine.

\section{Case study: energy-efficiency approach application}

The purpose of this case study is to optimize and simulate the energy-problem that would have been incurred if the water pumping stations and reservoirs are operated according to the Saskatoon West model developed in this paper, and to compare the optimized operational costs with the actual costs. In this work, the energy-efficiency approach is applied over a period of one week from $1^{\text {st }}$ to $7^{\text {th }}$ May 2011.

To overcome the problem of defining network boundary and operational conditions, it is important to plan pumping operations for more than one day, [12]. Planning pumping operations for multiple days is useful for systems with 
tanks that are large enough to hold significant storage volumes (e.g., in Saskatoon West water distribution system). In such situations, water can be stored when pumps are turned $\mathrm{ON}$, and the tanks can be emptied when pumps are turned OFF without affecting the consumption profile of the customers. Hence, for the Saskatoon system, results are obtained for 168 fixed time intervals of 1 hour.

For this case study, each run is conducted using the proposed AP-MODE and EPANET with a different randomly selected initial population of 100 candidate solutions. The parameters of the energy- problem considered in this work are based on technical characteristics of the pumping stations and reservoirs of the Saskatoon West water distribution system, and are described in Table 5. All experiments are run on an i7-3520M CPU (2.9 GHz) with 8 GB Ram using GNU/ WINDOWS 7.

Table 5: Technical characteristics and parameters of Saskatoon West system.

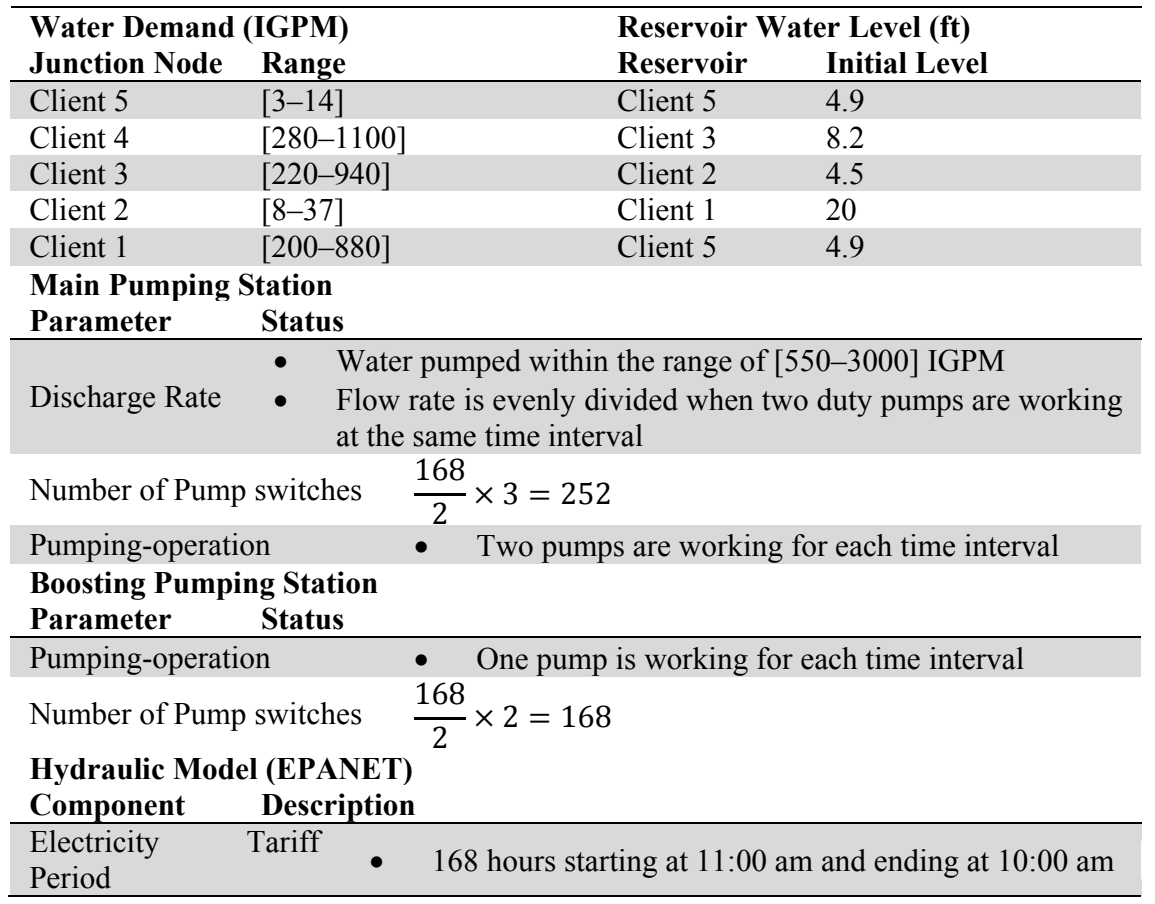

\section{Simulation solutions for the optimal operation}

An extensive analysis of the solutions obtained by the energy-efficiency approach strategy is carried out, examined, and compared in detail the outcome of each solution with regard to the cost function of the problem (i.e., five objectives). By means of statistical criterion, the configuration of the best 
solution found in each objective is obtained in which the least value pertaining to each objective is selected as a solution in this analysis. Amongst the solutions (i.e., there are 100 solutions obtained by the energy-efficiency approach when it is applied to the Saskatoon West system) provided, five solutions are then selected to evaluate and analyze the performance of the proposed approach. Moreover, these five solutions are then used to make the comparison and the discussion of the simulation results for this case study.

The discussion of the simulation results started first by comparing the best five solutions $(1,2,3,4$, and 5) with respect to their savings in energy costs. Table 6 presents the corresponding savings in both electrical energy cost and maximum demand charge compared to the actual costs measured for the first week of May 2011. In this table, the highest savings of $7.34 \%$, which is equivalent to a reduction of $\$ 243$ compared with the actual cost of $\$ 3315$, is obtained by solution 1 (i.e., the best solution with respect to the first objective). In the same table, solution 4 (i.e., the best solution with respect to the fourth objective) showed the lowest savings that is $4 \%$ (i.e., equivalent to $\$ 132$ ). With respect to the maximum demand charge, Table 6 shows that solution 2 (that is corresponding to the best solution for the second objective) carried out the highest savings of 5.14\% (i.e., equal to the demand charge of $\$ 1385$ compared with the actual charge of $\$ 1460$ ). On the other hand, solution 5 (i.e., the best solution with respect to the fifth objective) produced the lowest percentage savings of $3.7 \%$.

Table 6: Experimental results for the Saskatoon System - electrical energy costs.

Cost of Electrical Energy (\$)

\begin{tabular}{ccccccc}
$\begin{array}{c}\text { Solutio } \\
\mathbf{n}\end{array}$ & $\begin{array}{c}\text { Actual } \\
\text { Cost }\end{array}$ & $\begin{array}{c}\text { Optimize } \\
\text { d Cost }\end{array}$ & $\begin{array}{c}\text { Saving } \\
(\mathbf{\%})\end{array}$ & $\begin{array}{c}\text { Actual } \\
\text { Cost }\end{array}$ & $\begin{array}{c}\text { Optimize } \\
\text { d Cost }\end{array}$ & $\begin{array}{c}\text { Saving } \\
(\%)\end{array}$ \\
\hline 1 & 3315 & 3072 & 7.34 & 1460 & 1401 & 4.00 \\
2 & 3315 & 3163 & 4.56 & 1460 & 1385 & 5.14 \\
3 & 3315 & 3141 & 5.25 & 1460 & 1397 & 4.32 \\
4 & 3315 & 3182 & 4.00 & 1460 & 1399 & 4.18 \\
5 & 3315 & 3130 & 5.58 & 1460 & 1406 & 3.70 \\
\hline
\end{tabular}

The summary of the best five solutions are tabulated and presented in Table 7 . This table shows the values corresponding to the five objectives. For example, solution 4 produced the lowest reservoir level variation of $1.2 \mathrm{ft}, 85 \mathrm{pump}$ switches, and 2.5 IGPM difference in system mass beside the savings of energy costs of $4 \%$ and $4.18 \%$, respectively. While solution 1 produced savings in energy costs of $7.34 \%$ and $4 \%, 133$ pumps switches, $3.2 \mathrm{ft}$ water level variation, and over 3.4 IGPM difference in system mass. Unlike the single-objective optimization where only one optimal solution, in multi-objective optimization the conclusion that can be drawn herein is that there is no "the best solution" amongst those solutions, rather there is a set of high quality and approximated 
Table 7: $\quad$ Summary of experimental results.

\begin{tabular}{cccc}
\hline Solution & $\begin{array}{c}\text { Savings in Electricity } \\
\text { Cost (\%) }\end{array}$ & $\begin{array}{c}\text { Savings in Max. Demand } \\
\text { Charge (\%) }\end{array}$ & $\begin{array}{c}\text { Pump Switches } \\
\text { Range }^{*}\end{array}$ \\
\hline 1 & 7.34 & 4.00 & 133 \\
2 & 4.56 & 5.14 & 124 \\
3 & 5.25 & 4.32 & 142 \\
4 & 4.00 & 4.18 & 85 \\
5 & 5.58 & 3.70 & 127 \\
Solution & Reservoirs Level Variation & System Mass Difference (IGPM) \\
\hline 1 & $(\mathbf{f t})$ & & 3.4 \\
2 & 3.2 & & 3.7 \\
3 & 2.7 & & 3.1 \\
\hline 4 & 1.9 & & 2.5 \\
5 & -1.4 & & \\
\hline
\end{tabular}

*The total allowable pumps switches considered in this work is equal to 420 times.

**Small variations do not make solutions unacceptable [13].

optimal solutions. And therefore, based on the certain predefined criteria, the decision makers need to select one of these solutions most suited to their preferences to be implemented. Furthermore, this approach achieves a better optimal operation for water pumping stations and reservoirs in distribution systems, thereby leading to a more rational use of water and energy resources.

\section{Conclusion}

Operating pumping stations in water distribution systems requires significant amount of energy. Thus, a new energy-efficiency approach is developed to optimize pump scheduling and useful reservoir capacity that should be working to satisfy the water demand forecasting and system constraints at minimal operational cost. The energy-problem proposed in this paper is solved using a new multi-objective differential evolution with an adaptive technique, a parallelization mechanism, a parallel islands model, and a ring-network topology. The experimental results showed that the proposed energy-efficiency approach led into significant savings in the overall operational cost, and also led to considerable improvements in both system reliability and safety. It should be noted that all the obtained solutions are found to be feasible in terms of satisfying the system constraints, water demands, and hydraulic requirements. Furthermore, the energy-efficiency approach developed in this paper can be applied for any water system district, including irrigation, urban, rural, treated, and wastewater distribution systems. Finally, it is important to notice that in order to obtain accurate optimal pump scheduling and reservoir operation, the system should be well equipped with all types of sensors (i.e., power, pressure, water level, etc.), automatic control valves, switches, and supervisory control and 
data acquisition (SCADA). That is, this approach (and any other method of energy optimization) is highly related with the availability (i.e., quantitatively and qualitatively) information/data of the facilities.

\section{References}

[1] B. K. Mehta and A. Goto, "Design and Operation of On-farm Irrigation Ponds," Irrigation and Drainage Engineering, vol. 118, no. 5, pp. 659-673, 1992.

[2] D. Nel and J. Haarhoff, "Sizing Municipal Water Storage Tanks with the Monte Carlo Simulation," Water SRT-Aqua, vol. 45, no. 4, pp. 203-212, 1996.

[3] S. Hirose, "Determination of the Capacity of a Regulating Pond in a Pipeline Irrigation System," Rural Environmental Engineering, vol. 33, pp. 66-78, 1997.

[4] I. Pulido-Calvo, J. C. Gutiérrez-Estrada, R. López-Luque, and J. Roldán, "Regulating Reservoirs in Pressurized Irrigation Water Supply Systems," Water SRT-Aqua, vol. 55, no. 5, pp. 367-381, 2006 b.

[5] M. H. Sabet and O. J. Helweg, "Peaking Storage Tanks for Water Supply Systems," Water Resources, vol. 25, no. 5, pp. 1047-1053, 1989.

[6] E. Fred. and W. Larry, Water Distribution System Operation: Application of Simulated Annealing. Phoenix: Arizona State University, 2004.

[7] L. Mauel, T. Devi, and P. Ben, "Multi Objectives Optimization of the Pump Scheduling Problem Using SPEA2," Evolutionary Computation, IEEE Congress, pp. 435-442, 2005.

[8] T. M. Walski, et al., Advanced Water Distribution Modeling and Management. Waterbury, CT, USA: Bentley Institute Press, 2003.

[9] K. Price and R. Storn, "Differential Evolution - A Siimple Evolution Strategy for Fast Optimization," Software Tools for Professional Programmer, vol. 22, no. 4, p. 18-24, 1997.

[10] K. Price, R. Storn, and J. Lampinen, Differential Evolution - A Practical Approach to Global Optimization. Berlin, Germany: SpringerVerlag, 2005.

[11] E. Alba and J. Troya, "Analyzing Synchronous and Asynchronous Parallel Distributed Genetic Algorithms," Future Generation Computer Systems, 2001.

[12] K. E. Lansey and A. Kofi, “Optimal Pump Operation Considering Pump Switches," Water Resources Planning and Management, vol. 120, pp. 1-17, 1994.

[13] C. V. Lucken, B. Baran, and A. Sotelo, "Pump Scheduling Optimization Using Asynchronous Parallel Evolutionary Algorithms," CLEI ELECTRONIC, vol. 7, no. 2, pp. 40-60, Dec. 2004.

[14] D. E. Goldberg, Genetic Algorithms in Search Optimization and Machine Learning. Addison Wesley, 1989.

[15] M. Tomassini, "Parallel and Distributed Evolutionary Algorithms: A Review," Evolutionary Algorithms in Engineering and Computer Science, pp. 113-138, 1999. 